\title{
Contraceptive adherence among HIV-infected women in Malawi: a randomized controlled trial of the copper intrauterine device and depot medroxyprogesterone acetate ${ }^{\star,, k x}$
}

\author{
Lisa B. Haddad ${ }^{\mathrm{a},}$, Carrie Cwiak $^{\mathrm{a}}$, Denise J. Jamieson ${ }^{\mathrm{a}}$, Caryl Feldacker ${ }^{\mathrm{b}, \mathrm{c}}$, Hannock

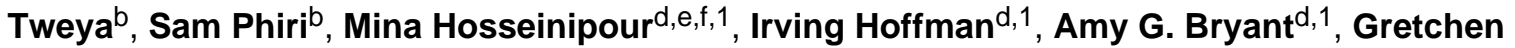 \\ S. Stuart ${ }^{\mathrm{d}, 1}$, Isaac Noah ${ }^{\mathrm{b}, 1}$, Linly Mulundila ${ }^{\mathrm{b}, 1}$, Bernadette Samala ${ }^{\mathrm{b}, 1}$, and Patrick Mayne ${ }^{\mathrm{g}, 1}$ \\ aEmory University, Department of Gynecology and Obstetrics, Atlanta, GA, USA \\ bThe Lighthouse Trust, Lilongwe, Malawi \\ CInternational Training and Education Center for Health (I-TECH), University of Washington, \\ Seattle, USA \\ dUniversity of North Carolina-Chapel Hill, Chapel Hill, NC, USA \\ eUNC-Project, Lilongwe, Malawi \\ ${ }^{f}$ Reproductive Health Services, Ministry of Health, Lilongwe, Malawi \\ gBrown University, Department of Sociology, Provence, RI, USA
}

\section{Abstract}

Objective-To evaluate contraceptive adherence to the copper intrauterine device (Cu-IUD) and the injectable depot medroxyprogesterone acetate (DMPA) among women with HIV in Lilongwe, Malawi.

\begin{abstract}
Methods-We randomized $200 \mathrm{HIV}$-infected women on HAART to either the Cu-IUD or DMPA and followed these women prospectively, evaluating adherence and factors associated with nonadherence.
\end{abstract}

\begin{abstract}
Results-There was no difference in contraceptive adherence: 68\% of Cu-IUD and 65\% of DMPA users were adherent at 48 weeks. Receiving first-choice contraceptive was not associated with adherence. Women commonly cited partner's disapproval as an indication for discontinuation. Women who experienced heavy menstruation and first-time contraceptive users were more likely to be nonadherent. Among ongoing users at study conclusion, $95 \%$ were happy with their method, and $98 \%$ would recommend their method to a friend.
\end{abstract}

\footnotetext{
Conflicts of interest: There are no conflicts of interest among any of the investigators or authors.

Sources of funding: This study was funded by an anonymous foundation and the center for AIDS research at Emory University. (C) 2013 Published by Elsevier Inc.

*Corresponding author. Tel.: +1 404778 1385; fax: +1 404778 1382. lbhadda@emory.edu (L.B. Haddad).

${ }^{1}$ Lighthouse UNC Emory Family Planning Study Group.
} 
Conclusion-Contraceptive adherence between the Cu-IUD and DMPA was similar at 1 year. With similar adherence and similar high rates of satisfaction among users of both methods at 1 year, the $\mathrm{Cu}$-IUD offers a hormone-free alternative to DMPA.

Implications-Adherence to the Cu-IUD and DMPA is similar at 1 year among HIV-infected women on HAART in a randomized controlled trial. Despite high method satisfaction, partner disapproval and heavy bleeding contribute to reduced adherence. Receiving a method that differs from participant's first-choice method did not influence adherence.

\section{Keywords}

HIV; Contraception; Adherence; IUD; Depot medroxyprogesterone acetate; DMPA

\section{Introduction}

Of the estimated 33 million people living with HIV/AIDS worldwide, 16 million are women [1]. Unsafe sexual practices in sub-Saharan Africa have generated high rates of HIV, sexually transmitted infections and unintended pregnancy. Specifically, preventing unintended pregnancy reduces maternal and child mortality, rates of mother-to-child transmission of HIV and poverty. Therefore, improving family planning services in these countries remains a key public health goal.

Although there is a high prevalence of injectable contraceptive use in many resource-limited settings, specifically depot medroxyprogesterone acetate (DMPA), continuation of these methods can be poor with studies reporting 12-month continuation of DMPA ranging from $23 \%$ to $78 \%$ [2-6]. Adherence to a contraceptive requires continuation of the method at appropriate intervals for efficacy. For DMPA, individuals must receive injections at 3-month intervals. In contrast, the copper intrauterine device (Cu-IUD) offers hormone-free, effective protection from pregnancy for up to 12 years. In Malawi, a large unmet need for family planning services remains. While contraceptive knowledge is almost universal, the contraceptive prevalence rate is $42 \%$ among married women using any form of modern birth control with only $0.2 \%$ of Malawian women using the intrauterine device (IUD) [7,8].

Research exploring contraceptive method adherence in sub-Saharan Africa and among HIVinfected women is lacking. Further, there are no studies on adherence among women receiving antiretroviral therapy (ART). With the increase of women receiving ART in Malawi with "Option B Plus" (initiation of lifelong ART among all HIV+ pregnant women) [9,10], understanding contraceptive use in this increasing population is critical. Women on ART have recurrent clinic visits for medication where contraceptives can be incorporated and thus adherence may be improved. Alternatively, these women may have other medical concerns or medication side effects that may influence contraceptive adherence. We sought to determine contraceptive adherence over 1 year with a randomized controlled trial (RCT) comparing the Cu-IUD and DMPA among HIV-infected women on ART in Malawi. Based on prior studies in developed [6] and developing countries [11], we hypothesized that among our HIV-positive cohort in Malawi, adherence for the Cu-IUD would be greater at 1 year than adherence to DMPA. Furthermore, we sought to determine factors associated with adherence, satisfaction, side effects and adverse events associated with these methods. 


\section{Methods}

HIV+ women seeking family planning services were enrolled by study staff into an RCT comparing the copper T380A IUD (Cu-IUD) to DMPA at the Lighthouse Clinic in Lilongwe, Malawi. Prior to study implementation, comprehensive family planning provision was established [12]. In Malawi, contraceptives are offered free of charge through the Ministry of Health.

Women attending their ART visits who were interested in family planning were referred to the study and assessed for their interest and eligibility. Eligibility criteria included: 18-45 years of age; HIV-positive status on ART for 6 months or longer; medical eligibility to receive the Cu-IUD and DMPA according to World Health Organization (WHO) criteria [13], Malawi National Reproductive Health Service Delivery Guidelines and clinical assessment; desire to delay pregnancy for 12 months or longer; and a plan to stay in the region for study duration. Genital tract infections were diagnosed and treated per Malawi sexually transmitted infections guidelines [14] using a syndromic management approach. The study protocol was approved by the Malawi National Health Science Research Council and institutional review boards at Emory University and the University of North Carolina at Chapel Hill (ClinicalTrials.gov identifier NCT01191203).

Women were randomly assigned to receive the Cu-IUD or DMPA at enrollment. The randomization occurred in blocks of four and six using an online randomizer (http:// www.randomizer.org/form.htm) with allocations placed in sequentially sealed envelopes prior to study initiation. Method allocation was not masked. One incorrect allocation to DMPA resulted in 99 women receiving the IUD and 101 women receiving DMPA.

At enrollment, participants completed a questionnaire, had a complete physical examination and received the allocated method. Follow-up visits were at 4, 12, 24, 36 and 48 weeks. At all follow-up visits, a questionnaire assessing side effects, a pregnancy test and physical examination were administered. At initial, 24- and 48-week visits, hemoglobin level was determined; at initial and 48-week visit, CD4 count was collected. At each visit, male and female condoms were provided and use encouraged. A bar of soap was given at each visit. No other financial incentives were offered.

Statistical analyses were performed using STATA version 11[15]. The primary outcome was contraceptive adherence. With 200 participants, we had an $80 \%$ power of detecting a $20 \%$ difference in adherence to the Cu-IUD compared to DMPA at 1 year with a two-sided $95 \%$ confidence interval (CI), assuming 20\% loss to follow-up. We defined adherence for the $\mathrm{Cu}$ IUD as the length of continuous time with the $\mathrm{Cu}$-IUD in place. If a $\mathrm{Cu}$-IUD expulsion was replaced at a visit, the individual was considered adherent. If the device was not replaced then nonadherence was noted at that time. If $\mathrm{Cu}$-IUD removal was requested, nonadherence was noted at the time of removal. For DMPA, adherence was defined as the length of continuous time between initial injection and 15 weeks after final injection [16,17]. The individual was nonadherent if DMPA was administered beyond 15 weeks from prior injection, if the subject declined DMPA, became pregnant or had a hysterectomy. 
Nonadherence was considered a single event. Individuals lost to follow-up were censored at the last visit with the $\mathrm{Cu}$-IUD in place or 15 weeks after the last DMPA injection.

The Kaplan-Meier method was used to estimate the probability of adherence. Differences in adherence between the DMPA and IUD groups were tested with log-rank tests. Cox proportional hazards models were used to estimate hazard ratios and CIs for adherence. A multivariate model was refined with backwards elimination removing variables that did not change the estimate by more than $10 \%$. Differences in the frequency of side effects were assessed using chi-square or Fisher's Exact Tests. Paired $t$ tests assessed changes in hemoglobin or CD4 count.

\section{Results}

A total of 281 women were screened between August 2010 and January 2011; 81 (28.8\%) were ineligible or refused enrollment: 12 (14.8\%) did not meet medical eligibility, 21 (25.9\%) were on ART for less than 6 months and 48 (59.3\%) desired a specific contraceptive method. There were no differences between mean age, gravidity and parity between enrolled and unenrolled. Of 200 women enrolled, mean age was $32.3(\mathrm{SD}=5.6$, median $=32$, range $=18-48)$, gravidity of $3.7(\mathrm{SD}=1.8$, median $=4.0$, range $=0-11)$ and parity of $3.3(\mathrm{SD}=1.8$, median $=4$, range $=0-11)$ (Table 1). At enrollment, 10 women reported current hormonal contraceptive use: nine DMPA users (two assigned to DMPA and seven assigned to IUD) and one pill user (assigned DMPA).

Overall, $133(66.5 \%)$ women were adherent at 48 weeks following contraceptive initiation, and cumulative adherence was similar between DMPA and Cu-IUD groups (Fig. 1). Of 99 $\mathrm{Cu}$-IUD users, 67 were adherent at 48 weeks, 29 women chose removal, 1 died and 2 were lost to follow-up. There were nine $\mathrm{Cu}$-IUD expulsions; three replacements and six declining replacement. Of 101 women who received DMPA, 66 were adherent at 48 weeks, 21 chose to discontinue, 9 were late for injections, 1 had a hysterectomy, 2 became pregnant, 1 died and 2 were lost to follow-up (Fig. 2).

The most common reasons for discontinuing the allocated method were side effects (19 women=11 Cu-IUD, 8 DMPA), partner's request ( 12 women=9 Cu-IUD, 3 DMPA), no longer being sexually active ( 6 women $=2 \mathrm{Cu}$-IUD, 4 DMPA) and desire to get pregnant ( 4 women=2 $\mathrm{Cu}$-IUD, 2 DMPA). One IUD removal was performed in the operating room via dilation and curettage.

After multivariate adjustment, method was still not significantly associated with adherence (Table 2). However, heavy menstruation during the study increased the likelihood of nonadherence $(\mathrm{HR}=2.6,95 \% \mathrm{CI}=1.06-6.36)$ while prior hormonal contraceptive experience decreased the likelihood of nonadherence ( $\mathrm{HR}=0.51,95 \% \mathrm{CI}=0.31-0.85)$. Notably, the concordance of the allocated method with the participant's first-choice method was not associated with adherence. Furthermore, there was no interaction between method received and first-choice method in a stratified analysis: among women receiving their first choice, the hazard ratio for adherence was $1.00(95 \% \mathrm{CI}=0.5-1.97)$ for DMPA and $1.63(95 \%$ $\mathrm{CI}=0.72-3.33$ ) for IUD. 
Among those adherent to the method at the last visit, satisfaction was not significantly different by method: $95 \%$ stated that they were happy with their method, and $98 \%$ would recommend it to a friend. For those who discontinued, $96 \%$ and $58 \%$ reported being happy with their contraceptive, and $94 \%$ and $81 \%$ would recommend their method to a friend on the visit prior to discontinuation and at their discontinuation visit, respectively.

$\mathrm{Cu}-\mathrm{IUD}$ use was significantly associated with heavy menstrual flow and cramps. DMPA was significantly associated with amenorrhea and reductions in libido (Table 3). The mean CD4 count at baseline and 48 weeks was unchanged for both groups ( $\mathrm{p}=.56): 430.1$ cells $/ \mathrm{mm}^{3}(\mathrm{SD}=245.0)$ and 464.2 cells $/ \mathrm{mm}^{3}(\mathrm{SD}=242.4)$ for the IUD group and 550.1 cells $/ \mathrm{mm}^{3}(\mathrm{SD}=237.5)$ and 599.5 cells $/ \mathrm{mm}^{3}(\mathrm{SD}=241.2)$ for the DMPA group. Hemoglobin at baseline and 48 weeks was unchanged for both groups ( $\mathrm{p}=.33$ ). There was no significant difference in the number of women who changed their ART regimen over the course of the study $(\mathrm{p}=.40)$.

\section{Discussion}

In this RCT, we demonstrated that the Cu-IUD offers an acceptable, hormone-free alternative to DMPA for HIV-positive women, with similar rates of adherence, high satisfaction and safety among users at 1 year. Our findings seem to challenge those from an RCT in Zambia [18] among HIV-infected women where 51\% of the Cu-IUD group and $87 \%$ of the hormonal contraceptive group were continuing at 2 years. This reported that high hormonal contraceptive continuation may overestimate the actual method adherence rate. However, our findings differ from those among healthy US women in which $\mathrm{Cu}$-IUD continuation was greater than for DMPA at 1 year (84\% vs. 57\%) [6]. Differences in adherence in the US may be due to contraceptive experience as we found familiarity with contraception was important for increased method adherence. Furthermore, HIV-positive women may have different health concerns than noninfected women impacting adherence.

Prior studies note lower adherence to the $\mathrm{Cu}$-IUD related to patient misconceptions about safety, provider discomfort, lack of social marketing and culture-specific concerns [19,20]. As DMPA has been the most common contraceptive used in Malawi while the IUD is just now being reintroduced, misconceptions about the IUD are likely to have a greater impact in this setting. Although we did not provide standardized counseling during enrollment or follow-up, providers reviewed common side-effects, misconceptions and management strategies with the goal of reducing method discontinuations. Future efforts need to address patient knowledge, community misconceptions, and social-cultural beliefs regarding contraception, specifically the IUD.

Reasons for nonadherence to either method in our study were similar to those reported among healthy and HIV-positive women in developing countries [5,21,22], including menstrual irregularities, desire for fertility or no current sexual activity [21,23-25]. Partners' desire to discontinue contraception was also common. Our counseling did not routinely include partners. Several women also discontinued due to side effects, some of which may not have been related to their method as evidenced by one patient who attributed generalized illness to her IUD. Overall, side effects were rare and similar to common reported side 
effects. Counseling women specifically about expected bleeding changes is important since heavy bleeding was a predictor of nonadherence. Notably, there was no difference in hemoglobin at 1 year despite increased bleeding reported among IUD users.

Adverse events were uncommon. Although one woman in each group died during the study follow-up, these were unlikely related to the contraceptive method. Two women became pregnant on DMPA; yet, based on the estimated gestational ages at follow-up, these pregnancies were likely conceived before DMPA became effective. The relatively high IUD expulsion rate may have been associated with provider inexperience; providers began placing IUDs concordant with study initiation. Although one woman needed to go to the operating room for IUD removal, it was the missing string and limited provider experience that led to hospital referral. One woman in the DMPA group had a hysterectomy, although we were unable to determine the reason.

Our study has several limitations. First, we do not measure adherence past 1 year. Cu-IUD adherence may improve with time compared to DMPA, supported by our findings of fewer IUD discontinuations after 36 weeks compared to DMPA. Second, there are baseline differences between the intervention arms with those in the IUD group having higher education and lower rates of unintended pregnancy. However, controlling for these factors did not impact rates of adherence or our conclusions.

A strength is that our primary endpoint was contraceptive adherence, defined as consistent and correct use of a method, rather than method continuation which may underestimate the impact of inconsistent use on contraceptive effectiveness. Evaluating adherence provides a better estimate of actual time of contraceptive exposure and protection from unintended pregnancy. We used 15 weeks as a cut off for adherence based on the WHO family planning provider handbook [17], the basic guidance for practice in Malawi. This may be an underestimate of DMPA effectiveness as evidence suggests that efficacy can extend for 17 weeks after an injection [16].

Another strength is the RCT design that removes potential patient selection and provider bias within a population where the IUD is not commonly used and where differential beliefs and health concerns could impact outcomes. Randomization in contraceptive studies is a topic of debate in the family planning community. It is posited that method choice may be a strong predictor of adherence and patient satisfaction such that randomization leads to a discontinuation bias. We did not find this to be the case as contraceptive choice was not associated with adherence. Overall method satisfaction was high, even among women discontinuing, highlighting that discontinuation is not always due to method disapproval. It is posited that the sample willing to be randomized may not be representative of the population; however, most eligible patients were enrolled and similar to unenrolled. Our results provide a good representation of contraceptive adherence in similar settings where family planning is integrated into HIV care.

Recently, renewed concerns surfaced over the potential for increased transmission of HIV and disease progression with hormonal contraception use [26,27]. However, not all studies support this link $[28,29]$, and methodological limitations across so few studies limit possible 
implications of the data thus far [30]. The WHO concluded that there was insufficient evidence to support a change in the current guidelines on the use of hormonal contraceptives [31]; yet, they encouraged further investigation on the impact of hormonal contraception and HIV risk, along with increased promotion of consistent use of condoms and other nonhormonal contraceptives. Demonstrating the feasibility and acceptability of an RCT and similar adherence to the copper IUD among HIV-infected women can assist future research efforts.

Among women with HIV, many were willing to accept a Cu-IUD for birth control as well as participate in an RCT for contraception. The Cu-IUD may have many benefits that support its promotion in sub-Saharan Africa where HIV is endemic, and we found that adherence to the Cu-IUD was similar to DMPA among HIV-infected women at 1 year. As multiple factors impact method adherence, including partner's desire to discontinue, altering fertility intentions and side effects, educational efforts need to address patient, provider and community misconceptions related to family planning.

\section{References}

1. UNAIDS. UNAIDS Report on the global AIDS epidemic. 2010.

2. Westfall JM, Main DS, Barnard L. Continuation rates among injectable contraceptive users. Fam Plan Perspect. 1996; 28:275-7.

3. Sangi-Haghpeykar H, Poindexter AN 3rd, Bateman L, Ditmore JR. Experiences of injectable contraceptive users in an urban setting. Obstet Gynecol. 1996; 88:227-33. [PubMed: 8692507]

4. Smit J, Beksinska M. Hormonal contraceptive continuation and switching in South Africa: Implications for evaluating the association of injectable hormonal contraceptive use and HIV. J Acquir Immune Defic Syndr. 2012

5. Nanda K, Morrison CS, Kwok C, et al. Discontinuation of oral contraceptives and depot medroxyprogesterone acetate among women with and without HIV in Uganda Zimbabwe and Thailand. Contraception. 2011; 83:542-8. [PubMed: 21570552]

6. Peipert JF, Zhao Q, Allsworth JE, et al. Continuation and satisfaction of reversible contraception. Obstet Gynecol. 2011; 117:1105-13. [PubMed: 21508749]

7. Malawi Ministry of Health. Malawi Demographic and Health Survey (MDHS). 2010.

8. Macro. NSONaI. Malawi Demographic and Health Survey 2010. Zomba, Malawi and Calverton Maryland, USA: NSO and ICF Macro; 2011.

9. Chinkonde-Nkhoma, JR.; Hosseinipour, M.; Mofolo, I., et al. A review of the 'Option B Plus' rollout in the five districts of Malawi. AIDS 2012: International AIDS conference; Washington DC, USA. 2012.

10. Tippett Barr, B.; Mhango, E.; Tenthani, L., et al. Uptake and retention in Malawi's Option B+ PMTCT program: Lifelong ART for all HIV+ pregnant or lactating women. 20th Conference on Retroviruses and Opportunistic Infections (CROI); Atlanta, GA, USA. 2013.

11. Ali, MM.; Sadler, RK.; Cleland, J.; Ngo, TD.; Shah, IH. Long-term contraceptive protection, discontinuation and switching behaviour: intrauterine device (IUD) use dynamics in 14 developing countries. London: World Health Organization and Marie Stopes International; 2011.

12. Haddad, L.; Nyirenda, M.; Tweya, H., et al. Successful integration of family planning into HIV care in Lilongwe, Malawi. Rome, Italy: International AIDS Society; 2011.

13. World Health Organization. Medical Eligibility Criteria for contraceptive use. 4. 2009.

14. Ministry of Health Malawi, National AIDS Commision and World Health Organization. Management of sexually transmitted infections using a syndromic management approach Guidelines for service providers. 6. 2007.

15. StataCorp. Stata Statistical Software: Release 11. College Station, TX: StataCorp LP; 2009. 
16. Hatcher, R.; Trussell, J.; Nelson, A.; Cates, W.; Stewart, F.; Kowel, D. Contraceptive technology. 20. New York, N.Y: Ardent Media; 2011.

17. World Health Organization Department of Reproductive Health and Research (WHO/RPR) and Johns Hopkins Bloomberg School of Public Health/Center for Communication Programs (CCP). Family Planning: A Global Handbook for Providers. Baltimore and Geneva: CCP and WHO; 2007.

18. Stringer EM, Kaseba C, Levy J, et al. A randomized trial of the intrauterine contraceptive device vs hormonal contraception in women who are infected with the human immunodeficiency virus. Am J Obstet Gynecol. 2007; 197:144e1-8. [PubMed: 17689627]

19. Stanwood NL, Garrett JM, Konrad TR. Obstetrician-gynecologists and the intrauterine device: a survey of attitudes and practice. Obstet Gynecol. 2002; 99:275-80. [PubMed: 11814509]

20. van Zijl SMC, van der Spuy ZM. A survey to assess knowledge and acceptability of the intrauterine device in the family planning services in Cape Town, South Africa. J Fam Plann Reprod Health Care. 2010; 36:73-8. [PubMed: 20406549]

21. Barden-O'Fallon J, Speizer I, Rodriguez F, Calix J. Experience with side effects among users of injectables, the IUD, and oral contraceptive pills in four urban areas of Honduras. Health Care Women Int. 2009; 30:475-83. [PubMed: 19418321]

22. Tolley E, Loza S, Kafafi L, Cummings S. The impact of menstrual side effects on contraceptive discontinuation: findings from a longitudinal study in Cairo, Egypt. Int Fam Plan Perspect. 2005; 31:15-23. [PubMed: 15888405]

23. Ali MM, Cleland J. Contraceptive switching after method-related discontinuation: levels and differentials. Stud Fam Plann. 2010; 41:129-33. [PubMed: 21466113]

24. Ali MM, Cleland J. Oral contraceptive discontinuation and its aftermath in 19 developing countries. Contraception. 2010; 81:22-9. [PubMed: 20004269]

25. Sunyavivat S, Chumnijarakij T, Onthuam Y, Mehyar AH, Busca B, Christian B. Reasons for discontinuing contraception among women in Bangkok. Bull World Health Organ. 1983; 61:8615. [PubMed: 6606502]

26. Heffron R, Donnell D, Rees H, et al. Use of hormonal contraceptives and risk of HIV-1 transmission: a prospective cohort study. Lancet Infect Dis. 2011

27. Wand H, Ramjee G. The effects of injectable hormonal contraceptives on HIV seroconversion and on sexually transmitted infections. AIDS. 2012; 26:375-80. [PubMed: 22156970]

28. Reid SE, Dai JY, Wang J, et al. Pregnancy, contraceptive use, and HIV acquisition in HPTN 039: relevance for HIV prevention trials among African women. J Acquir Immune Defic Syndr. 2010; 53:606-13. [PubMed: 19838129]

29. Morrison CS, Skoler-Karpoff S, Kwok C, et al. Hormonal contraception and the risk of HIV acquisition among women in South Africa. AIDS. 2012; 26:497-504. [PubMed: 22156973]

30. Blish CA, Baeten JM. Hormonal contraception and HIV-1 transmission. Am J Reprod Immunol. 2011; 65:302-7. [PubMed: 21087338]

31. World Health Organization (WHO). Hormonal contraception and HIV Technical Statement. Feb 16. 2012 Available at http://whqlibdoc.who.int/hq/2012/WHO_RHR_12.08_eng.pdf 


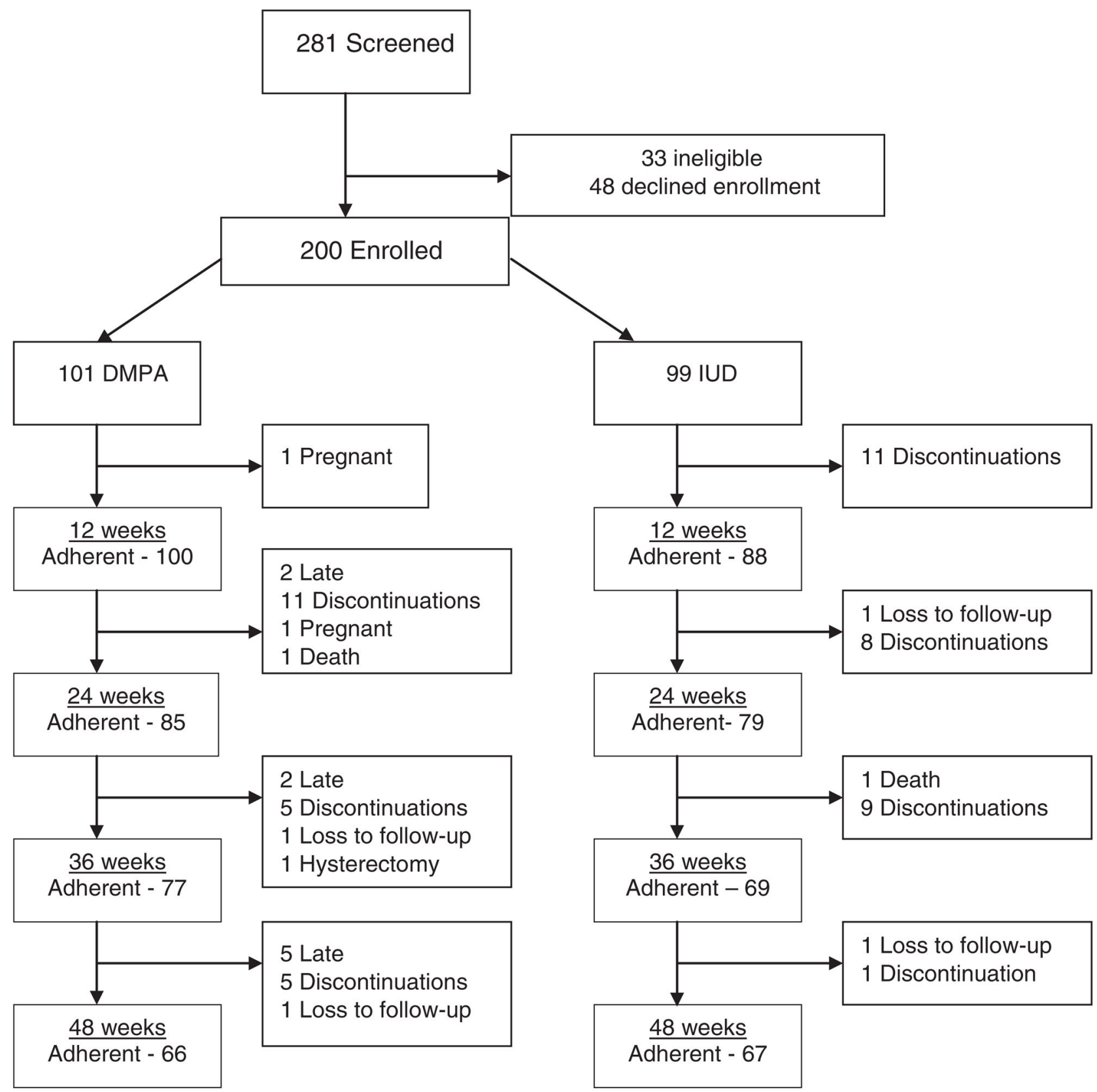

Fig. 1.

Unadjusted Kaplan-Meier survival curve of adherence over time by study arm ( $\mathrm{p}=.919)$. 


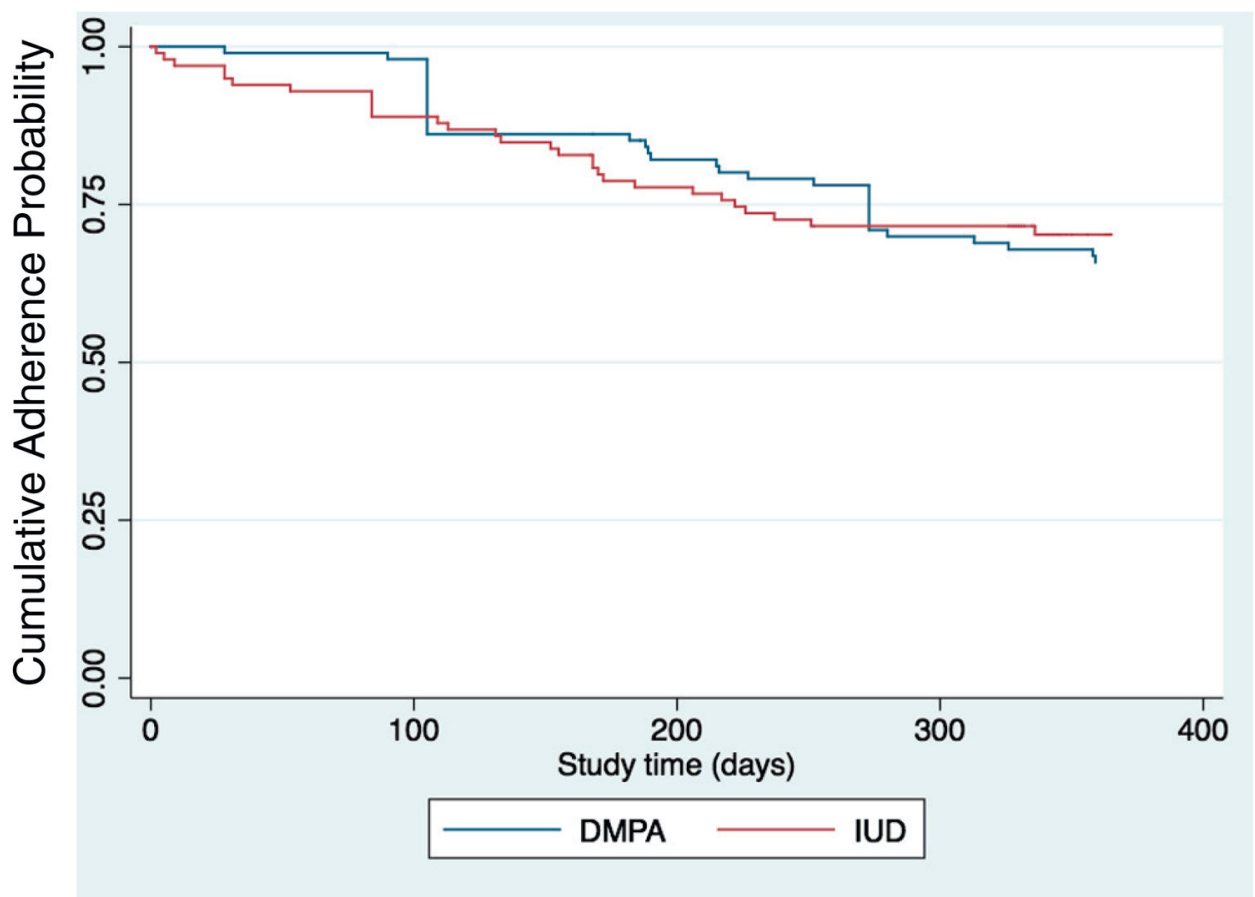

Fig. 2.

Study screening, enrollment and followup over 48 weeks. Cumulative adherence probability. 


\section{Table 1}

Baseline characteristics by study arm

\begin{tabular}{|c|c|c|c|}
\hline \multirow[t]{2}{*}{ Variable } & \multirow{2}{*}{$\frac{\text { DMPA }(n=101)}{\operatorname{Count}(\%)}$} & \multirow{2}{*}{$\frac{\text { IUD }(n=99)}{\operatorname{Count}(\%)}$} & \multirow[t]{2}{*}{ p Value } \\
\hline & & & \\
\hline Concordance between first-choice method and assigned method & $57(56.4)$ & $43(43.4)$ & .066 \\
\hline Age at enrollment [mean (SD)] & $32.21(5.22)$ & $32.44(6.03)$ & .767 \\
\hline \multicolumn{4}{|l|}{ Education } \\
\hline Less than primary completed (reference) & $55(54.5)$ & $39(39.4)$ & $.048^{*}$ \\
\hline Primary completed but less than secondary completed & $31(30.7)$ & $47(47.5)$ & \\
\hline Some education beyond secondary & $14(13.9)$ & $13(13.1)$ & \\
\hline Last pregnancy unintentional & $77(76.2)$ & $60(60.6)$ & $.046^{*}$ \\
\hline Ever had an abortion & $32(31.7)$ & $23(23.2)$ & .226 \\
\hline \multicolumn{4}{|l|}{ Number of past pregnancies } \\
\hline 0 or 1 (reference) & $7(6.9)$ & $10(10.1)$ & .421 \\
\hline 2 or more & $94(93.1)$ & $89(89.9)$ & \\
\hline \multicolumn{4}{|l|}{ Partners in the past year } \\
\hline 0 & $3(3.0)$ & $6(6.1)$ & .555 \\
\hline 1 & $92(91.1)$ & $88(88.9)$ & \\
\hline 2 or more & $6(5.9)$ & $5(5.1)$ & \\
\hline \multicolumn{4}{|l|}{ Relationship status } \\
\hline Not currently living with partner (reference) & $23(22.8)$ & $30(30.3)$ & .227 \\
\hline Currently living with partner & $78(77.2)$ & $69(69.7)$ & \\
\hline Partner desires more children & $9(8.9)$ & $5(5.1)$ & .362 \\
\hline \multicolumn{4}{|l|}{ Partner support for birth control } \\
\hline Partner does not support (reference) & $8(7.9)$ & $5(5.1)$ & .553 \\
\hline Partner supports & $77(76.2)$ & $74(74.7)$ & \\
\hline Partner opinion unknown & $16(15.8)$ & $20(20.2)$ & \\
\hline \multicolumn{4}{|l|}{ Partner HIV status } \\
\hline HIV positive (reference) & $56(55.5)$ & $51(51.5)$ & .853 \\
\hline HIV negative & $10(9.9)$ & $11(11.1)$ & \\
\hline Partner status unknown & $35(34.7)$ & $37(37.4)$ & \\
\hline Previously used hormonal birth control method ${ }^{a}$ & $71(70.3)$ & $68(68.7)$ & .805 \\
\hline Heavy menstruation at baseline & $3(3)$ & $3(3)$ & .980 \\
\hline
\end{tabular}




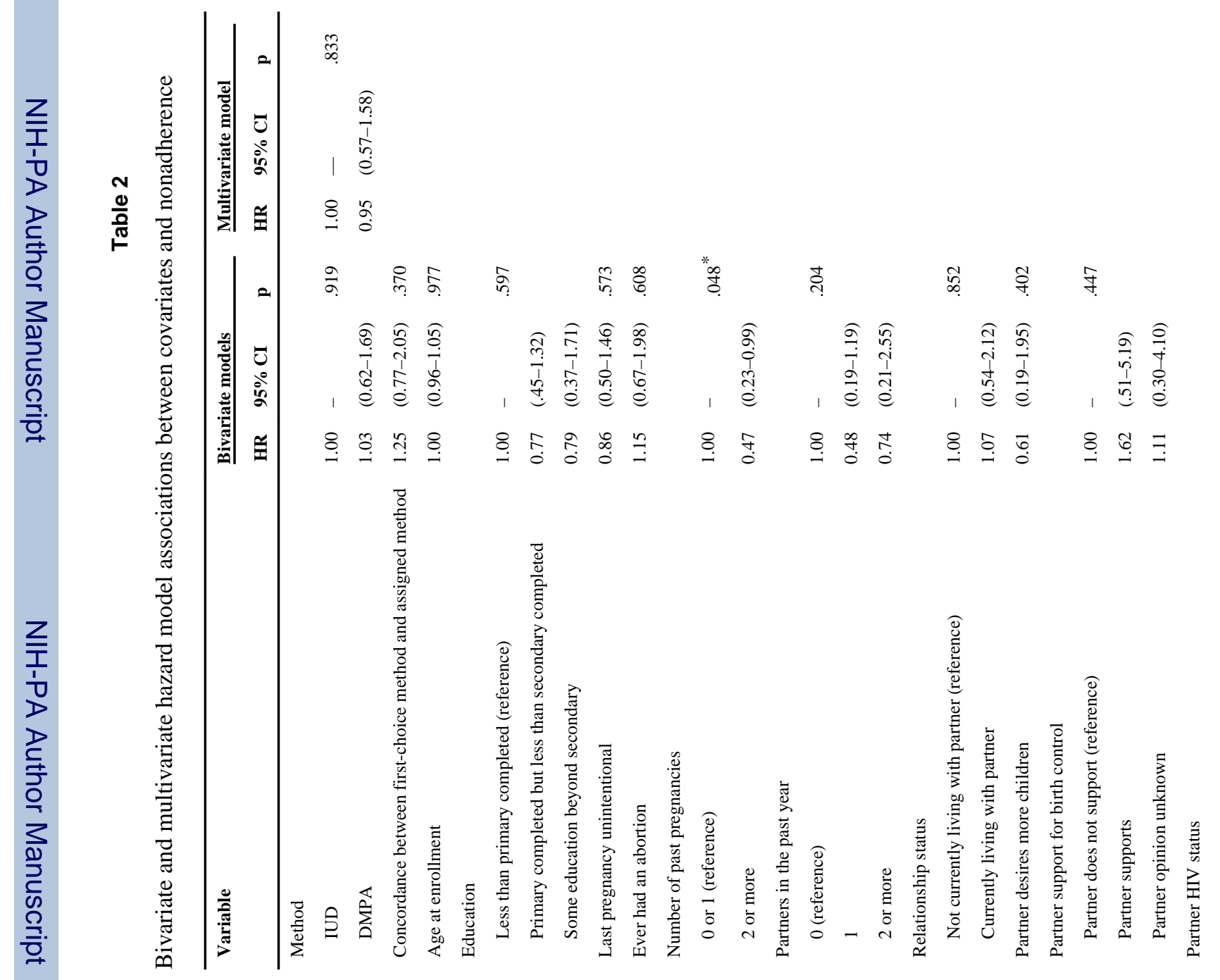




\section{Table 3}

Side effects by treatment arm

\begin{tabular}{lcccl}
\hline Variable & IUD & DMPA & p \\
\cline { 2 - 2 } & $\boldsymbol{n}(\boldsymbol{\eta})$ & $\boldsymbol{n}(\boldsymbol{\%})$ & \\
\hline Heavy menstrual flow & $18(18.2)$ & $8(7.9)$ & $.031^{*}$ \\
Spotting & $22(22.2)$ & $32(31.6)$ & .133 \\
Amenorrhea & $55(55.6)$ & $70(69.4)$ & $.045^{*}$ \\
Cramps & $19(19.2)$ & $6(5.9)$ & $.005^{*}$ \\
Headache & $27(27.3)$ & $30(29.8)$ & .704 \\
Dizziness & $17(17.2)$ & $13(12.8)$ & .395 \\
Nausea & $15(15.2)$ & $18(17.8)$ & .611 \\
Breast tenderness & $5(5.1)$ & $9(8.9)$ & .287 \\
Weight gain & $57(57.6)$ & $58(57.4)$ & .982 \\
Weight loss & $49(49.5)$ & $37(36.6)$ & .067 \\
Depression & $12(12.1)$ & $9(8.9)$ & .461 \\
Reduction in libido & $31(31.3)$ & $52(51.4)$ & $.004^{*}$ \\
Pain during sex & $10(10.1)$ & $12(11.8)$ & .688 \\
Abdominal pain & $27(27.3)$ & $17(16.8)$ & .075 \\
Vaginal discharge & $3(3.0)$ & $8(7.9)$ & .226 \\
Backache & $31(31.3)$ & $30(29.8)$ & .805 \\
Hair loss & $6(6.1)$ & $3(3)$ & .478 \\
\hline * & & & \\
p<.05. & & & \\
& & &
\end{tabular}

